\title{
On Bernstein Type Inequalities and a Weighted Chebyshev Approximation Problem on Ellipses
}

\author{
Roland Freund
}

May, 1989

Research Institute for Advanced Computer Science

NASA Ames Research Center

RLACS Technical Report 89.21

NASA Cooperative Agreement Number NCC 2-387
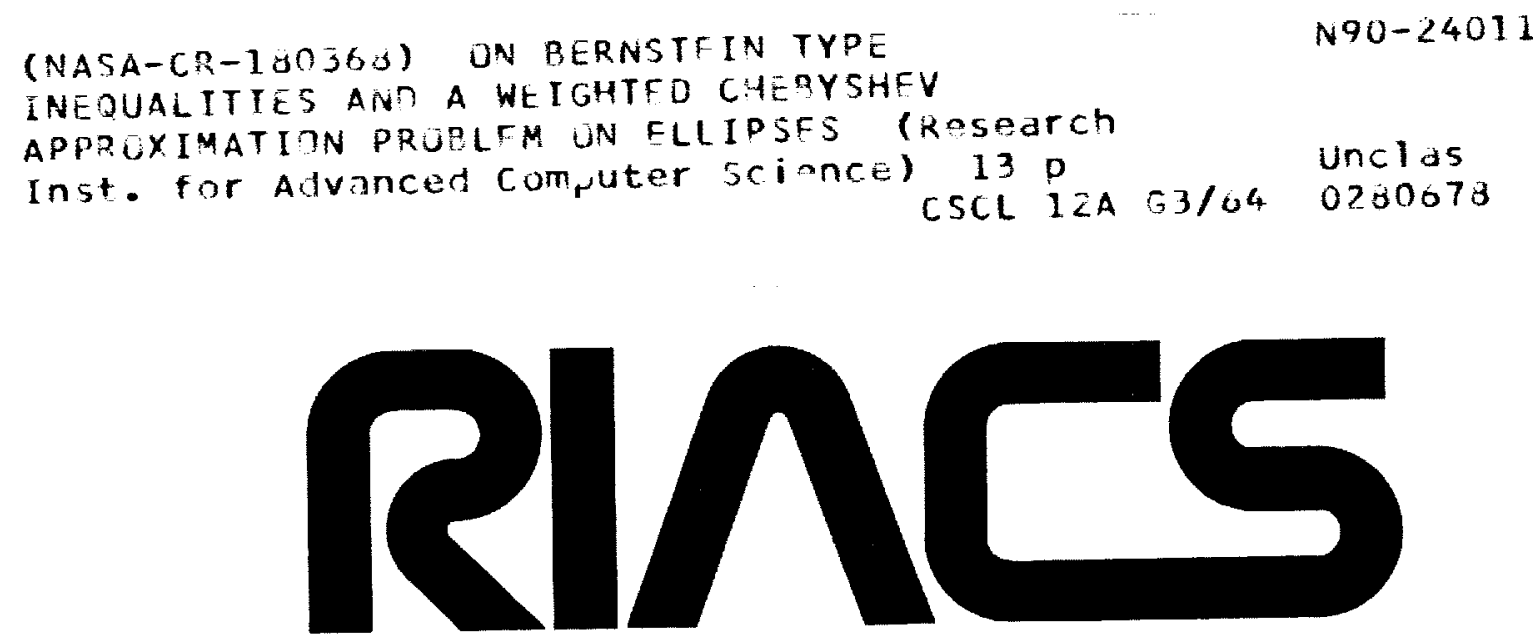

Research Institute for Advanced Computer Sclence 


\title{
On Bernstein Type Inequalities and a Weighted Chebyshev Approximation Problem on Ellipses
}

\author{
Roland Freund
}

May, 1989

Research Institute for Advanced Computer Science NASA Ames Research Center

RIACS Technical Report 89.21

NASA Cooperative Agreement Number NCC 2-387 
- 


\title{
On Bernstein Type Inequalities and a Weighted Chebyshev Approximation Problem on Ellipses
}

\author{
Roland Freund \\ Institut für Angewandte Mathematik und Statistik \\ Universität Würzburg \\ Am Hubland \\ D - 8700 Würzburg \\ Federal Republic of Germany \\ and \\ RIACS, Mail Stop 230-5 \\ NASA Ames Research Center \\ Moffett Field, CA 94035, USA
}

\begin{abstract}
We are concerned with a classical inequality due to Bernstein which estimates the norm of polynomials on any given ellipse in terms of their norm on any smaller ellipse with the same foci. For the uniform and a certain weighted uniform norm, and for the case that the two ellipses are not ${ }^{d}$ too close", we derive sharp estimates of this type and determine the corresponding extremal polynomials. These Bernstein type inequalities are closely connected with certain constrained Chebyshev approximation problems on ellipses. We also present some new results for a weighted approximation problem of this type.
\end{abstract}

\section{Introduction}

Let $\Pi_{n}$ denote the set of all complex polynomials of degree at most $n$. For $r \geq 1$, let

$$
\mathcal{E}_{r}:=\left\{z \in \mathbf{C}|| z-1|+| z+1 \mid \leq r+\frac{1}{r}\right\}
$$

be the ellipse with foci at \pm 1 and semi-axes $(r \pm 1 / r) / 2$. Moreover, we use the notation $\|\cdot\| \mathcal{E}_{r}$ for the uniform norm $\|f\|_{\mathcal{E}_{r}}=\max _{z \in \mathcal{E}_{r}}|f(z)|$ on $\mathcal{E}_{r}$.

It is well known (see e.g. [8, Problem III. 271, p. 137]) that, for any $n \in \mathbb{N}$ and $R>r \geq 1$

$$
\|p\|_{\mathcal{E}_{R}} \leq \frac{R^{n}}{r^{n}}\|p\|_{\mathcal{E}_{r}} \text { for all } p \in \Pi_{n}
$$

This work was supported by Cooperative Agreement NCC 2-387 between the National Aeronautics and Space Administration (NASA) and the Universities Space Research Association (USRA) 
We remark that for the case $r=1, \mathcal{E}_{1}=[-1,1]$, this inequality goes back to Bernstein (see [2] and the references therein). It is also well known (see [12, p. 368]) that the estimate (2) is not sharp, i.e. equality in (2) holds only for the trivial polynomial $p \equiv 0$.

In this note, we are mainly concerned with the following two problems: find the best possible constants $C_{n}(r, R)$ and $C_{n+1 / 2}(r, R)$ such that

$$
\|p\|_{\mathcal{E}_{R}} \leq C_{n}(r, R)\|p\|_{\mathcal{E}_{r}} \text { for all } p \in \Pi_{n}
$$

and

$$
\|w p \mid\|_{\mathcal{E}_{R}} \leq C_{n+1 / 2}(r, R)\|w p\|_{\mathcal{E}_{r}} \quad \text { for all } \quad p \in \Pi_{n},
$$

respectively. Here, and in the sequel, $w$ denotes the weight function $w(z)=\sqrt{z+1}$, and it is always assumed that the square root is chosen such that $w$ maps the $z$-plane onto $\{\operatorname{Re} w>0\} \cup\{i \eta \mid \eta \geq 0\}$. We notice that the usual proof (e.g. [8, p. 320]) for (2) immediately carries over to the weighted case (4) and leads to the upper bound $C_{n+1 / 2}(r, R)<R^{n+1 / 2} / r^{n+1 / 2}$. For the classical case $r=1$, Frappier and Rahman [2] conjectured that

$$
C_{n}(1, R)=\frac{1}{2}\left(R^{n}+R^{n-2}\right) \quad \text { and } \quad C_{n+1 / 2}(1, R)=\frac{1}{2}\left(R^{n+1 / 2}+R^{n-3 / 2}\right) .
$$

The first identity in (5) was proved in [9] for $n=1$ and in [4] for $n=2, R \geq \sqrt{3}$. The second relation in (5) is known to be true for $n=1$ and $R \geq 1.49$ [4]. It seems that these are the only cases for which the best possible constants in (3) and (4) are known.

In this paper, sharp estimates (3) and (4) will be obtained for the case $n \in \mathbb{N}$, $r>1$, and $R$ not "too close" to $r$. More precisely, we will prove the following

Theorem 1. Let $n \in \mathbb{N}$ and $r>1$.

a) If

$$
R \geq r \frac{73 r^{4}-1}{r^{4}-1} \quad \text { resp. } \quad R \geq r \frac{33 r-1}{r-1},
$$

then

$$
C_{n}(r, R)=\frac{R^{n}+1 / R^{n}}{r^{n}+1 / r^{n}} \quad \text { resp. } \quad C_{n+1 / 2}(r, R)=\frac{R^{n+1 / 2}+1 / R^{n+1 / 2}}{r^{n+1 / 2}+1 / r^{n+1 / 2}}
$$

with equality holding in (3) resp. (4) only for the polynomials

$$
p(z) \equiv \gamma\left(T_{n}(z)+i \frac{2 \delta}{R^{n}-1 / R^{n}}\right), \gamma \in C, \delta \in[-1,1] \quad \text { resp. } \quad p(z) \equiv \gamma V_{n}(z), \gamma \in C .
$$

Moreover, if $n=1$, the first identity in (7) holds true for all $R>r$.

b)

$$
C_{n}(r, R) \geq \frac{R^{n}+1 / R^{n}}{r^{n}+1 / r^{n}} \quad \text { and } \quad C_{n+1 / 2}(r, R) \geq \frac{R^{n+1 / 2}+1 / R^{n+1 / 2}}{r^{n+1 / 2}+1 / r^{n+1 / 2}}
$$


for all $R>r$.

In (8) and in the following, the notation $T_{k}$ is used for the $k$ th Chebyshev polynomial which by means of the Joukowsky map is given by

$$
T_{k}(z) \equiv \frac{1}{2}\left(v^{k}+\frac{1}{v^{k}}\right) \quad, \quad z \equiv \frac{1}{2}\left(v+\frac{1}{v}\right),
$$

and, moreover,

$$
V_{k}(z) \equiv \frac{T_{k+1}(z)+T_{k}(z)}{\sqrt{2}(z+1)} .
$$

Notice that (10) defines indeed a polynomial of degree $k$, and that $V_{k}$ is up to a scalar factor just the $k$ th Jacobi polynomial $p_{k}^{(-1 / 2,1 / 2)}$ associated with the weight function $(1-z)^{-1 / 2}(1+z)^{1 / 2}$ on $[-1,1]$ (cf. Szegö $[13$, p. 60]).

Remark 1. The estimates (6) are very crude in the following sense. Let $R_{n}(r)$ resp. $R_{n+1 / 2}(r)$ denote the smallest numbers such that the first resp. the second identity in (7) is satisfied for all $R \geq R_{n}(r)$ resp. $R \geq R_{n+1 / 2}(r)$. Numerical tests reveal that $R_{n}(r)$ and $R_{n+1 / 2}(r)$ are much smaller than the upper bounds in (6). Moreover, these experiments suggest that $R_{n}(r), R_{n+1 / 2}(r) \approx r$ for large $n$. However, we were not able to prove these numerical observations.

Although the weighted norms in (4) might appear somewhat artificial, note that (4) arises naturally if, using the Joukowsky map (cf. (9)), one rewrites the estimates (3) and (4) for the disks $|v| \leq R,|v| \leq r$, and the class of self-reciprocal polynomials

$$
\Sigma_{m}:=\left\{s \in \Pi_{m} \mid v^{m} s(1 / v) \equiv s(v)\right\}
$$

(cf. $[2,4,5])$. More precisely,

$$
\begin{aligned}
v^{n} p\left(\frac{1}{2}\left(v+\frac{1}{v}\right)\right) \equiv s(v), \quad p \in \Pi_{n}, \quad s \in \Sigma_{2 n}, \\
\text { resp. } \quad v^{n+1 / 2} w\left(\frac{1}{2}\left(v+\frac{1}{v}\right)\right) p\left(\frac{1}{2}\left(v+\frac{1}{v}\right)\right) \equiv s(v), \quad p \in \Pi_{n}, \quad s \in \Sigma_{2 n+1},
\end{aligned}
$$

defines a one-to-one mapping between $\Pi_{n}$ and $\Sigma_{2 n}$ resp. $\Sigma_{2 n+1}$. With (11), it is easily verified that (3), (4) are equivalent to

$$
\max _{|v| \leq R}|s(v)| \leq D_{m}(r, R) \max _{|v| \leq r}|s(v)| \text { for all } s \in \Sigma_{m},
$$

where (3) and (4) correspond to the case $m=2 n$ and $m=2 n+1$, respectively. Moreover, the best possible constants in (3), (4), and (12) are connected by $D_{m}(r, R)=$ $(R / r)^{m / 2} C_{m / 2}(r, R)$. Rewriting Theorem 1 for (12), then yields the following

Corollary. Let $m \geq 2$ be an integer and $r>1$.

a) If

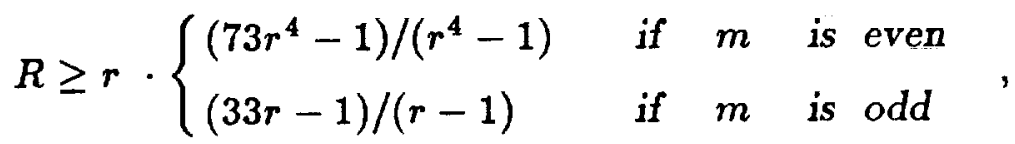

then

$$
D_{m}(r, R)=\frac{R^{m}+1}{r^{m}+1}
$$


with equality holding in (12) only for the polynomials

$s(v) \equiv \gamma\left(v^{2 n}+i \frac{4 \delta}{R^{n}-1 / R^{n}} v^{n}+1\right), \quad \gamma \in C, \delta \in[-1,1], \quad$ if $\quad m=2 n$ is even

and

$$
s(v) \equiv \gamma\left(v^{m}+1\right), \quad \gamma \in C, \quad \text { if } m \text { is odd . }
$$

Moreover, $D_{2}(r, R)=\left(R^{2}+1\right) /\left(r^{2}+1\right)$ for all $R>r$.

b)

$$
D_{m}(r, R) \geq \frac{R^{m}+1}{r^{m}+1} \quad \text { for all } R>r
$$

Our proof of Theorem 1 is based on the obvious representations

$$
C_{n}(r, R)=\max _{c \in \partial \mathcal{E}_{R}} \frac{1}{E_{n}(r, c)} \quad \text { and } \quad C_{n+1 / 2}(r, R)=\max _{c \in \partial \mathcal{E}_{R}} \frac{1}{E_{n+1 / 2}(r, c)}
$$

of the sharp constants in (3) and (4) in terms of the optimal values of the family of constrained Chebyshev approximation problems

$$
\left(E_{n}(r, c):=\min _{p \in \Pi_{n}: p(c)=1}\|p\|_{\mathcal{E}_{r}}\right.
$$

and

$$
\left(E_{n+1 / 2}(r, c):=\right) \min _{p \in \Pi_{n}: p(c)=1}\left\|w_{c} p\right\| \mathcal{E}_{r} \quad \text { where } \quad w_{c}(z) \equiv w(z) / w(c)
$$

Here, $c \in \mathbb{C} \backslash \mathcal{E}_{r}$ in (14) and (15), and, in (13), $\partial \mathcal{E}_{R}$ denotes the boundary of $\mathcal{E}_{R}$. The class of complex approximation problems (14) was investigated recently by Fischer and Freund [3]. In particular, the part of Theorem 1 which is concerned with the Bernstein type inequality (3) will follow from results given in [3]. In the present note, we will also derive some new results for the weighted variant (15).

The outline of the paper is as follows. In Section 2, we establish some auxiliary results. In Section 3, the complex weighted approximation problem (15) is studied. Finally, remaining proofs are given in Section 4.

\section{Preliminaries}

In this section, we introduce some further notations and list two lemmas. It will be convenient to define, in analogy to the Chebyshev polynomials (9), the functions

$$
T_{k+1 / 2}(z) \equiv \frac{1}{2}\left(v^{k+1 / 2}+\frac{1}{v^{k+1 / 2}}\right), \quad z \equiv \frac{1}{2}\left(v+\frac{1}{v}\right) \quad, \quad k=0,1, \ldots .
$$

Here the square root $\sqrt{v}$ is chosen, correspondingly to $w(z)=\sqrt{z+1}$, such that $\sqrt{v}$ maps the $v$-plane onto $\{\operatorname{Re} \xi>0\} \cup\{i \eta \mid \eta \geq 1$ or $-1<\eta \leq 0\}$ (cf. [4]). With (10) and (16), one readily verifies that then

$$
T_{k+1 / 2}(z)=w(z) V_{k}(z), \quad z \in \mathbb{C},
$$


holds.

For the boundary points $z \in \partial \mathcal{E}_{r}$ of the ellipse (1), we will use the parametrization

$$
z=z_{r}(\varphi)=\frac{1}{2}\left(r+\frac{1}{r}\right) \cos \varphi+\frac{i}{2}\left(r-\frac{1}{r}\right) \sin \varphi \quad, \quad-\pi<\varphi \leq \pi .
$$

With (16) and (18), it follows that

$$
T_{k+1 / 2}\left(z_{r}(\varphi)\right)=a_{k} \cos \left(k+\frac{1}{2}\right) \varphi+i b_{k} \sin \left(k+\frac{1}{2}\right) \varphi \quad, \quad-\pi<\varphi \leq \pi,
$$

where

$$
a_{k}:=\frac{1}{2}\left(r^{k+1 / 2}+\frac{1}{r^{k+1 / 2}}\right) \quad \text { and } \quad b_{k}:=\frac{1}{2}\left(r^{k+1 / 2}-\frac{1}{r^{k+1 / 2}}\right) .
$$

Next, assume that $n \in \mathbb{N}$ and $r>1$. Using (17) and (19), we deduce that

$$
\left\|w V_{n}\right\|_{\varepsilon_{\mathrm{r}}}=a_{n}
$$

All corresponding extremal points $z_{l} \in \mathcal{E}_{r}$, defined by $\left|w\left(z_{l}\right) V_{n}\left(z_{l}\right)\right|=\left\|w V_{n}\right\|_{\mathcal{E}_{r}}$, are given by

$$
z_{l}:=z_{r}\left(\varphi_{l}\right) \quad, \quad \varphi_{l}:=\frac{2 l \pi}{2 n+1} \quad, \quad l=-n,-n+1, \ldots, n-1, n .
$$

Moreover, we note that, in view of (17), (19), and (22),

$$
V_{n}\left(z_{l}\right)=(-1)^{l} \frac{a_{n}}{w\left(z_{l}\right)} \quad, \quad l=-n, \ldots, n .
$$

The following property of the numbers $\varphi_{l}$ will be used in the next section.

Lemma 1. Let $j \in \mathbb{Z}$. Then:

$$
\sum_{l=-n}^{n}(-1)^{l} e^{i \varphi_{l}\left(j+\frac{1}{2}\right)}=\left\{\begin{array}{ll}
2 n+1 & \text { if } \frac{2 j+1}{2 n+1} \in 2 \mathbb{Z}+1 \\
0 & \text { otherwise }
\end{array} .\right.
$$

Proof. With $q:=(2 j+1) /(2 n+1)$, we have $e^{i \varphi_{l}(j+1 / 2)}=\left(e^{q \pi i}\right)^{l}$. If $q \in 2 \mathbb{Z}+1$, then $e^{q \pi i}=-1$, and (24) is obviously true in this case. For $q \notin 2 \mathbb{Z}+1,(24)$ follows from

$$
\sum_{l=-n}^{n}\left(-e^{q \pi i}\right)^{l}=\left(-e^{q \pi i}\right)^{-n} \frac{1-\left(-e^{q \pi i}\right)^{2 n+1}}{1+e^{q \pi i}}
$$

and $\left(-e^{q \pi i}\right)^{2 n+1}=1$.

Finally, we will apply the following result due to Rogosinski and Szegö [11] in Section 4.

Lemma 2. Let $\lambda_{0}, \lambda_{1}, \ldots, \lambda_{n}$ be real numbers which satisfy $\lambda_{n} \geq 0, \lambda_{n-1}-2 \lambda_{n} \geq 0$, and $\lambda_{k-1}-2 \lambda_{k}+\lambda_{k+1} \geq 0$ for $k=1,2, \ldots, n-1$. Then:

$$
t(\varphi):=\frac{\lambda_{0}}{2}+\sum_{k=1}^{n} \lambda_{k} \cos (k \varphi) \geq 0 \text { for all } \varphi \in \mathbb{R} .
$$




\section{Results for the weighted approximation problem (15)}

In this section, we are concerned with the constrained Chebyshev approximation problem (15). In the sequel, it is assumed that $n \in \mathbb{N}, r \geq 1$, and $c \in \mathbf{C} \backslash \mathcal{E}_{\mathrm{r}}$. Standard results from approximation theory (see e.g. [6]) then guarantee that there always exists a unique optimal polynomial for (15). For the case $r=1$ of the unit interval $\mathcal{E}_{1}=[-1,1]$ and $c \in \mathbb{R} \backslash[-1,1]$, Bernstein [1] proved that the rescaled polynomial (10)

$$
v_{n}(z ; c) \equiv \frac{V_{n}(z)}{V_{n}(c)}
$$

is the extremal function for (15). For purely imaginary $c$ and, again, $r=1$, Freund and Ruscheweyh [4] showed that the optimal polynomial is a suitable linear combination of $v_{n}, v_{n-1}$, and $v_{n-2}$. To the best of our knowledge, these two cases seem to be the only ones for which the solution of (15) is explicitly known.

For the rest of the paper, we assume that $r>1$. It turns out that, somewhat surprisingly, (26) is also best possible for the general class (15) with complex $c$ as long as $c$ is not "too close" to $\mathcal{E}_{r}$. For the following, it will be convenient, to represent $c \notin \mathcal{E}_{r}$ in the form

$$
c=c_{r}(\psi)=\frac{1}{2}\left(R+\frac{1}{R}\right) \cos \psi+\frac{i}{2}\left(R-\frac{1}{R}\right) \sin \psi \quad, \quad R>r,-\pi<\varphi \leq \pi .
$$

In analogy to (19) and (20), it follows that

$$
d_{k}:=T_{k+1 / 2}(c)=A_{k} \cos \left(k+\frac{1}{2}\right) \psi+i B_{k} \sin \left(k+\frac{1}{2}\right) \psi
$$

where

$$
A_{k}:=\frac{1}{2}\left(R^{k+1 / 2}+\frac{1}{R^{k+1 / 2}}\right) \quad \text { and } \quad B_{k}:=\frac{1}{2}\left(R^{k+1 / 2}-\frac{1}{R^{k+1 / 2}}\right) .
$$

Based on Rivlin and Shapiro's characterization [10] of the optimal solution of general linear Chebyshev approximation problems, we next derive a simple criterion for the polynomial (26) to be best possible in (15). Note that the extremal points of $v_{n}(z ; c)$ are just the $z_{l}, l=-n, \ldots, n$, stated in (22). By applying the theory [10] to (15), (26), and by using (23), we obtain the following

Criterion. $v_{n}(z ; c)$ is the unique optimal polynomial for (15) iff there exist nonnegative real numbers $\sigma_{-n}, \sigma_{-n+1}, \ldots, \sigma_{n}$ (not all zero) such that

$$
\sum_{l=-n}^{n} \sigma_{l}(-1)^{l} w\left(z_{l}\right) q\left(z_{l}\right)=0 \text { for all } q \in \Pi_{n} \text { with } q(c)=0 \text {. }
$$

Clearly, it suffices to check (30) for the polynomials

$$
q(z) \equiv V_{k}(z)-V_{k}(c) \quad, \quad k=1,2, \ldots, n .
$$


i

With (17) and (28), this leads to the following equivalent formulation of (30):

$$
\sum_{l=-n}^{n} \sigma_{l}(-1)^{l}\left(d_{0} T_{k+1 / 2}\left(z_{l}\right)-d_{k} T_{1 / 2}\left(z_{l}\right)\right)=0 \quad, \quad k=1,2, \ldots, n .
$$

It turns out that there are simple formulae for all real solutions $\sigma_{l}$ of $\left(30^{\prime}\right)$. The trick is to use the ansatz

$$
\sigma_{l}=\sum_{j=0}^{n}\left(\mu_{j} \cos \left(j \varphi_{l}\right)+\nu_{j} \sin \left(j \varphi_{l}\right)\right) \quad, \quad \varphi_{l}=\frac{2 l \pi}{2 n+1} \quad, \quad l=-n, \ldots, n
$$

where $\mu_{j}, \nu_{j} \in \mathbb{R}, j=0, \ldots, n$. Note that such a representation (31) is possible for any collection of $\sigma_{-n}, \ldots, \sigma_{n} \in \mathbb{R}$. Now we insert (31) into $\left(30^{\prime}\right)$ and rewrite $T_{k+1 / 2}\left(z_{l}\right)$ and $T_{1 / 2}\left(z_{l}\right)$ in the form (19). Then, a routine calculation, making repeatedly use of Lemma 1 , shows that $\left(30^{\prime}\right)$ reduces to the equations

$$
\mu_{k} a_{n-k}-i \nu_{k} b_{n-k}-\left(d_{n-k} / d_{0}\right)\left(\mu_{n} a_{0}-i \nu_{n} b_{0}\right)=0, k=1,2, \ldots, n-1,
$$

and

$$
2 \mu_{0} a_{n}-\left(d_{n} / d_{0}\right)\left(\mu_{n} a_{0}-i \nu_{n} b_{0}\right)=0 .
$$

By determining all real solutions $\mu_{k}, \nu_{k}$ of the linear system (32a,b) and with (31), one easily verifies that all real numbers satisfying $\left(30^{\prime}\right)$ are given by $\sigma_{l}=\tau \sigma_{l}^{\star}, l=-n, \ldots, n$ with $\tau \in \mathbb{R}$ arbitrary and $\sigma_{l}^{\star}$ defined in (33). Hence, in view of the Criterion, we have proved the following

Theorem 2. $v_{n}(z ; c)$ is the unique optimal polynomial in (15) iff the numbers

$$
\begin{gathered}
\sigma_{l}^{\star}:=\frac{1}{2} \frac{\left|d_{n}\right|^{2}}{a_{n}}+\sum_{k=1}^{n}\left(\frac{\operatorname{Re}\left(d_{n-k} \overline{d_{n}}\right)}{a_{n-k}} \cos \left(k \varphi_{l}\right)+\frac{\operatorname{Im}\left(d_{n-k} \overline{d_{n}}\right)}{b_{n-k}} \sin \left(k \varphi_{l}\right)\right), \\
l=-n,-n+1, \ldots, n-1, n,
\end{gathered}
$$

are either all nonnegative or all nonpositive. Here $a_{k}, b_{k}, d_{k}$, and $\varphi_{l}$ are defined in (20), (28), and (22).

The numbers (33) are positive whenever $R / r$ is sufficiently large. In particular, in the next section we will prove the following

Theorem 3. Let $c=c_{R}(\psi)$ with $R>r>1$ (cf. (27)). Then:

a)

$$
E_{n+1 / 2}(r, c) \leq \frac{r^{n+1 / 2}+1 / r^{n+1 / 2}}{\sqrt{\left(R^{n+1 / 2}+1 / R^{n+1 / 2}\right)^{2}-4 \sin ^{2}(n+1 / 2) \psi}} .
$$

b) If $R \geq r(33 r-1) /(r-1)$, then $v_{n}(z ; c)$ is the unique optimal polynomial for (15) and equality holds in (34).

For the case that $c$ in (15) is real, we have the following sharper result. 
Theorem 4. Let $r>1$ and $c \in \mathbb{R}$. If (i) $c \geq r+1 / r-1 / 2$ or (ii) $c \leq-r$, then $v_{n}(z ; c)$ is the unique optimal polynomial for (15) and

$$
E_{n+1 / 2}(r, c)=\left\{\begin{array}{ll}
\frac{r^{n+1 / 2}+1 / r^{n+1 / 2}}{R^{n+1 / 2}+1 / R^{n+1 / 2}} & \text { in case (i) } \\
\frac{r^{n+1 / 2}+1 / r^{n+1 / 2}}{R^{n+1 / 2}-1 / R^{n+1 / 2}} & \text { in case (ii) }
\end{array} .\right.
$$

Remark 2. In contrast to the case $r=1$, for $r>1$, the polynomial $v_{n}(z ; c)$ is not best possible in (15) for all $c \in \mathbb{R} \backslash \mathcal{E}_{r}$. Indeed, numerical tests show that among the corresponding numbers (33), in general, positive and negative $\sigma_{l}^{\star}$ occur if $c$ is very close to $\mathcal{E}_{r}$.

Finally, we note that Theorem 3 is analogous to the following result for the unweighted approximation problem (14).

Theorem A. (Fischer, Freund [3]). Let $c=c_{R}(\psi)$ with $R>r>1$ (cf. (27)). Then: a)

$$
E_{n}(r, c) \leq \frac{r^{n}+1 / r^{n}}{R^{n}+1 / R^{n}}
$$

b) If $R \geq r\left(73 r^{4}-1\right) /\left(r^{4}-1\right)$, then

$$
p_{n}(z ; c) \equiv \frac{\left(R^{n}-1 / R^{n}\right) T_{n}(z)+2 i \sin (n \psi)}{\left(R^{n}-1 / R^{n}\right) T_{n}(c)+2 i \sin (n \psi)}
$$

is the unique optimal polynomial for (14) and equality holds in (35).

Remark 3. For $n=1$, (14) was solved completely by Opfer and Schober [7]. From their result, one can deduce (see [3]) that, for the case $n=1$, the statement in part b) of Theorem $\mathrm{A}$ is true for all $R>r \geq 1$.

Clearly, in view of (13), Theorem 1 is an immediate consequence of Theorem 3 , Theorem A, and Remark 3. The proofs of Theorem 3 and 4 will be given in the next section.

\section{Proofs of Theorem 3 and 4}

Proof of Theorem 3. With (17) and (21), it follows that

$$
E_{n+1 / 2} \leq\left\|\frac{w(z) V_{n}(z)}{w(c) V_{n}(c)}\right\|_{\mathcal{E}_{r}}=\frac{a_{n}}{\left|T_{n+1 / 2}(c)\right|}
$$

By (20) and (28) (both with $k=n$ ), the right-hand side is just the upper bound in (34). We now turn to part b). Using (28) and (29), it follows that

$$
\begin{aligned}
& \left|\operatorname{Re}\left(d_{k} \overline{d_{n}}\right)\right| \leq A_{k} A_{n}+B_{k} B_{n} \leq R^{n+k+1}, \\
& \left|\operatorname{Im}\left(d_{k} \overline{d_{n}}\right)\right| \leq A_{k} B_{n}+B_{k} A_{n} \leq R^{n+k+1},
\end{aligned}
$$


and

$$
\left|d_{n}\right|^{2} \geq A_{n}^{2}-1 \geq \frac{1}{4} R^{2 n+1}\left(1-2 / R^{2 n+1}\right)
$$

Let $\sigma_{\imath}^{\star}$ be given by (33). With (36), (37), and (20), we obtain the lower bound

$$
\sigma_{l}^{\star} \geq \frac{1}{4}\left(\frac{R^{2}}{r}\right)^{n} \frac{R \sqrt{r}}{r+1}\left(1-\frac{2}{R^{2 n+1}}\right)-4 R^{n+1} \sqrt{r} \sum_{k=0}^{n-1}\left(\frac{R}{r}\right)^{k} \frac{r^{4 k+1}}{r^{4 k+2}-1}
$$

Now assume that $R \geq r(33 r-1) /(r-1)$. With

$$
1-\frac{2}{R^{2 n+1}} \geq \frac{1}{2} \quad \text { and } \quad \frac{r^{4 k+1}}{r^{4 k+2}-1} \leq \frac{r}{r^{2}-1}, k=0,1, \ldots,
$$

we deduce from (38) that

$$
\sigma_{l}^{\star} \geq \frac{1}{8}\left(\frac{R^{2}}{r}\right)^{n} \frac{R \sqrt{r}}{(r+1)(R-r)}\left(R-r-32 \frac{r^{2}}{r-1}\right) \geq 0 .
$$

In view of Theorem 2, this concludes the proof of Theorem 3.

Proof of Theorem 4. First we consider the case (i), i.e. assume that

$$
c=\frac{1}{2}\left(R+\frac{1}{R}\right) \geq r+\frac{1}{r}-\frac{1}{2} .
$$

Then $\psi=0$ in (28), and the representation (33) reduces to

$$
\sigma_{l}^{\star}=A_{n}\left(\frac{1}{2} \frac{A_{n}}{a_{n}}+\sum_{k=1}^{n} \frac{A_{n-k}}{a_{n-k}} \cos \left(k \varphi_{l}\right)\right), l=-n, \ldots, n .
$$

It follows that $\sigma_{l}^{\star}=A_{n} t\left(\varphi_{l}\right)$ where $t$ is the trigonometric polynomial (25) with

$$
\lambda_{0}:=\frac{A_{n}}{a_{n}} \quad \text { and } \quad \lambda_{k}=\frac{A_{n-k}}{a_{n-k}} \quad, \quad k=1, \ldots, n
$$

Therefore, Theorem 2 together with Lemma 2 implies that $v_{n}$ is best possible in (15) provided that the numbers (40) satisfy the assumptions of Lemma 2. Hence, it remains to verify that the estimates

$$
\frac{A_{1}}{a_{1}} \geq 2 \frac{A_{0}}{a_{0}} \text { and } \frac{A_{k+1}}{a_{k+1}}-2 \frac{A_{k}}{a_{k}}+\frac{A_{k-1}}{a_{k-1}} \geq 0, \quad k=1, \ldots, n-1,
$$

hold. It is easily seen that the first condition in (41) is equivalent to (39). A more lengthy, but straightforward, computation shows that (39) also guarantees that the remaining inequalities in (41) are satisfied. We omit the details.

For the case (ii), $c \leq-r$, one proceeds similarly. Now $\psi=\pi$ in (28), and from (33) we obtain

$$
\sigma_{l}^{\star}=B_{n}\left(\frac{1}{2} \frac{B_{n}}{a_{n}}+\sum_{k=1}^{n} \frac{B_{n-k}}{a_{n-k}} \cos k\left(\varphi_{l}+\pi\right)\right), l=-n, \ldots, n .
$$


By applying Lemma 2, this time with

$$
\lambda_{0}:=\frac{B_{n}}{a_{n}} \quad \text { and } \quad \lambda_{k}=\frac{B_{n-k}}{a_{n-k}} \quad, \quad k=1, \ldots, n,
$$

and Theorem 2, we conclude that $v_{n}$ is the optimal polynomial for (15) if the assumptions of Lemma 2 are satisfied. A lengthy computation shows that the condition $c \leq-r$ indeed implies that the numbers (42) fulfill the required inequalities. Again, details are omitted here.

Acknowledgement. The author would like to thank Dr. Bernd Fischer for performing some numerical experiments which were very helpful for developing the results of Section 3 .

\section{References}

[1] Bernstein, S.N.: Sur une classe de polynomes d'écart minimum. C. R. Acad. Sci. Paris 190, 237-240 (1930)

[2] Frappier, C., Rahman, Q.I.: On an inequality of S. Bernstein. Can. J. Math. 34, 932-944 (1982)

[3] Fischer, B., Freund, R.: On the constrained Chebyshev approximation problem on ellipses. J. Approx. Theory, to appear

[4] Freund, R., Ruscheweyh, St.: On a class of Chebyshev approximation problems which arise in connection with a conjugate gradient type method. Numer. Math. $48,525-542(1986)$

[5] Govil, N.K., Jain, V.K., Labelle, G.: Inequalities for polynomials satisfying $p(z) \equiv$ $z^{n} p(1 / z)$. Proc. Amer. Math. Soc. 57, 238-242 (1976)

[0] Meinardus, G.: Approximation of functions: Theory and numerical methods. Berlin, Heidelberg, New York: Springer 1967

[7] Opfer, G., Schober, G.: Richardson's iteration for nonsymmetric matrices. Linear Algebra Appl. 58, 343-361 (1984)

[8] Pólya, G., Szegö, G.: Aufgaben und Lehrsätze aus der Analysis, Vol. I. Berlin, Heidelberg, New York: Springer, 4th ed., 1970

[9] Rahman, Q.I.: Some inequalities for polynomials. Proc. Amer. Math. Soc. 56, 225-230 (1976)

[10] Rivlin, T.J., Shapiro, H.S.: A unified approach to certain problems of approximation and minimization. J. Soc. Indust. Appl. Math. 9, 670-699 (1961)

[11] Rogosinski, W., Szegö, G.: Über die Abschnitte von Potenzreihen, die in einem Kreise beschränkt bleiben. Math. Z. 28, 73-94 (1928)

[12] Smirnov, V.I., Lebedev, N.A.: Functions of a complex variable. London: Iliffe Books 1968 
[13] Szegö, G.: Orthogonal polynomials. Amer. Math. Soc. Colloq. Publ., Vol. 23. Providence, R. I.: Amer. Math. Soc., 4th ed., 1975 


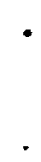

